\title{
The Application Value of ceMDCT in the Diagnosis of Gastric Cancer Extramural Vascular Invasion and Its Influencing Factors
}

\author{
Shifeng Yang, Xiaoming Zou (D), Jiacheng Li, Ange Zhang, Lei Zhu, Xiaolong Hu, \\ and Changjian Li \\ Department of Gastrointestinal Surgery, The Second Hospital of Harbin Medical University, Harbin, China \\ Correspondence should be addressed to Xiaoming Zou; zou4930@163.com
}

Received 20 October 2021; Revised 18 November 2021; Accepted 4 December 2021; Published 7 January 2022

Academic Editor: M. A. Bhagyaveni

Copyright (c) 2022 Shifeng Yang et al. This is an open access article distributed under the Creative Commons Attribution License, which permits unrestricted use, distribution, and reproduction in any medium, provided the original work is properly cited.

\begin{abstract}
Objective. To investigate the value of enhanced multislice spiral CT (ceMDCT) in the diagnosis of extramural vascular invasion of gastric cancer and the influencing factors of extramural vascular invasion. There are different methods used in this paper. Method. 131 patients with primary gastric cancer treated in our hospital from January 2017 to May 2019 were selected. All patients underwent surgical resection and ceMDCT examination before operation. Result. There were 40 cases with extramural vascular invasion of gastric cancer by surgical pathological diagnosis. The kappa value of ceMDCT in diagnosing extramural vascular invasion of gastric cancer was 0.947 , and the consistency was excellent. The diagnostic sensitivity, specificity, positive predictive value, and negative predictive value were $100.00 \%, 96.70 \%, 93.02 \%$, and $100.00 \%$, respectively. The proportions of T3-T4, tumour diameter $\geq 5.0 \mathrm{~cm}$, and growth pattern of proximal nodular + diffuse type in patients with gastric cancer extramural vascular invasion were $92.50 \%, 85.00 \%$, and $65.00 \%$, respectively, which were significantly higher than those in patients without extramural vascular invasion $(P<0.05)$. The logistic regression analysis results showed that T3-T4, tumour diameter $\geq 5.0 \mathrm{~cm}$, proximal nodular + diffuse growth pattern were the risk factors for extrahepatic vascular invasion in gastric cancer $(\mathrm{OR}=3.751,2.901$, and 3.367, $P<0.05)$. Conclusion. ceMDCT has good application value in diagnosing gastric cancer extramural vascular invasion. The occurrence of gastric cancer extramural vascular invasion is affected by T staging, tumour diameter, and tumour growth pattern.
\end{abstract}

\section{Introduction}

Gastric cancer is a malignant tumour originating from the epithelium of the gastric mucosa and is the most common of all malignant tumours of the digestive tract [1]. Gastric cancer has the characteristics of unobvious early symptoms and poor prognosis. Patients are already in the middle and late stages of gastric cancer when they are diagnosed, and the treatment is difficult. The most common clinical treatment is surgical resection of the lesion [2]. Extramural vascular invasion is a common complication of gastric cancer, which refers to the distant spread of the tumour into veins outside the gastrointestinal muscular layer, with an incidence rate of about 30\% [3]. It is important to judge whether the patient has extramural vascular invasion before surgery. Because of the different experiences of doctors, the judgment of extravascular invasion is not the same, and the judgment error is also significant. It is not appropriate to use pathology to judge the occurrence of extravascular invasion. Contrastenhanced multislice spiral CT (ceMDCT) can provide highresolution images and multiplanar reconstruction techniques. Preoperative imaging technology can diagnose the vascular wall invasion of gastric cancer. Clinically, a comprehensive analysis of ceMDCT imaging and pathology is needed to improve the diagnostic efficacy of the two. This study selected 131 patients with primary gastric cancer treated in our hospital from January 2017 to May 2019 to explore the application value of ceMDCT in the diagnosis of extramural vascular invasion of gastric cancer and its influencing factors. 
TABLE 1: Comparison of ceMDCT and surgical pathological results.

\begin{tabular}{lcccc}
\hline \multirow{2}{*}{ ceMDCT diagnosis } & \multicolumn{2}{c}{$\begin{array}{c}\text { Surgical pathology } \\
\text { There is no extracellular vascular invasion }\end{array}$} & \multirow{2}{*}{ Kappa } & $P$ \\
& Therawall vascular invasion & There is & 3 & \\
There is extrawall vascular invasion & 40 & 88 & 0.947 & $0.01^{*}$ \\
There is no extracellular vascular invasion & 0 & 8 & \\
\hline
\end{tabular}

$P<0.05$ indicates that the difference is statistically significant.

\section{Data and Methods}

2.1. General Information. 131 patients with primary gastric cancer treated in our hospital from January 2017 to May 2019 were selected, including 73 males and 58 females; age $21-70$ years, median age $(51.03 \pm 7.22)$ years; and pathological type: 61 cases of squamous cell carcinoma, 39 cases of adenosquamous carcinoma, and 31 cases of papillary adenocarcinoma. Inclusion criteria: (1) pathologically confirmed; (2) ceMDCT examination and surgical treatment in our hospital; (3) TNM staging refers to the standards in the 8th edition of the TNM staging system for gastric cancer issued by UICC and AJCC [4]; and (4) complete preservation of clinical data. Exclusion criteria: (1) with other malignant tumours; (2) poor image quality; and (3) preoperative radiotherapy and chemotherapy treatment history.

2.2. ceMDCT Inspection. Scanning and reconstruction parameters: fasting $8 \mathrm{~h}$ before the examination, drinking $600 \sim 800 \mathrm{ml} 5 \mathrm{~min}$ before the examination, and moderate filling of the gastric cavity. 64-row MDCT (GE USA) was used. MDCT scan parameters were $120 \mathrm{kV}, 240-260 \mathrm{mAs}$, collimation 64-0.6, and slice thickness $5 \mathrm{~mm}$, ranging from the diaphragm to pubic symphysis. After a plain scan, the high-pressure syringe (Ulrich $\mathrm{GmbH} \& \mathrm{Co}$, Germany) was used for the injection of $100 \mathrm{ml}$ iodine contrast agent (iopromide, German Bayer Xianling Pharmaceutical Company) through the median cubital vein at the injection rate of $2.5 \mathrm{ml} / \mathrm{s}$, the action phase, and portal vein scan. The original image of the portal phase was reconstructed at transverse, sagittal, and coronal positions using the Advantage Workstation 4.3 (GE, USA). The thickness of the reconstructed image layer was $1.25 \mathrm{~mm}$.

2.3. Pathological Examination. During the operation, the doctor observed whether the tumour spread with the naked eye, judged the invasion of extravascular blood vessels, and compared it with the preoperative judgment for statistical calculation.

2.4. Statistical Treatment. IBM SPSS Statistics 22.0 (SPSS Inc., Chicago, IL, USA) was used for statistical analysis. The experimental data were presented as ACCEPTED MANUSCRIPT mean \pm standard deviation (mean \pm SD), and the $t$-test was used for count data. The $\chi 2$ test and kappa test were used with kappa value $>0.75$ and excellent consistency; multivariate analysis used logistic regression analysis. One-way analysis of variance was used for comparison of parametric data among groups, followed by Turkey's test for comparison between two groups, while the Mann-Whitney $U$ test was performed for difference analysis of nonparametric data, and $P<0.05$ indicates that the difference is statistically significant.

\section{Results}

3.1. Surgical Pathologic Results. After surgical pathological diagnosis, out of 131 patients with gastric cancer, 40 had an external vascular invasion of gastric cancer.

3.2. ceMDCT Diagnostic Results. The consistent kappa value of c eMDCT diagnostic extrawall vascular invasion and surgical pathology outcome of gastric cancer was 0.947 , with its diagnostic sensitivity, specificity, and positive and negative predicted values of $100.00 \%(40 / 40), 96.70 \%(88 / 91)$, $93.02 \%(40 / 43)$, and $100.00 \%(88 / 88)$, respectively, as shown in Table $1, P=0.01$.

3.3. Comparison of Clinical Data of Patients with or without Extramural Vascular Invasion of Gastric Cancer. The group's example number, gender, age, and $\mathrm{T}, \mathrm{N}$, and $\mathrm{M}$ installment are all shown in Tables 2 . We can inform that T3 T4 tumours with gastric cancer had a significantly higher proximal nodular type + proportion than those with nonvalvular vascular invasion $(P<0.05)$.

3.4. Multifactor Analysis. Logistic regression analysis of $\mathrm{T}$ stage, tumour diameter, and growth mode as the independent variables and whether gastric cancer wall vascular invasion occurred as the dependent variable showed that T3 T4, tumour diameter $\geq 5.0 \mathrm{~cm}$, and proximal nodular + diffuse growth mode were risk factors for extrawall vascular invasion in gastric cancer $(\mathbf{O R}=3.751,2.901$, and 3.367, $P<0.05$ ), as shown in Table 3 .

\section{Discussion}

Gastric cancer is a common malignant tumour occurring in the digestive tract. It has the characteristics of minor early clinical symptoms and poor patient prognosis. The survival rate of patients with early gastric cancer is very high. Studies have shown that the survival rate of early gastric cancer is more than $90 \%$, and the survival rate of advanced gastric cancer is far lower than that of patients with early gastric cancer [5]. The incidence of vascular invasion outside the wall of the common complications of gastric cancer is about $30 \%$. Still, because the patient's clinical performance is not obvious, the condition is not easy to diagnose, resulting in the patient's condition gradually aggravating due to the delay and even reducing 


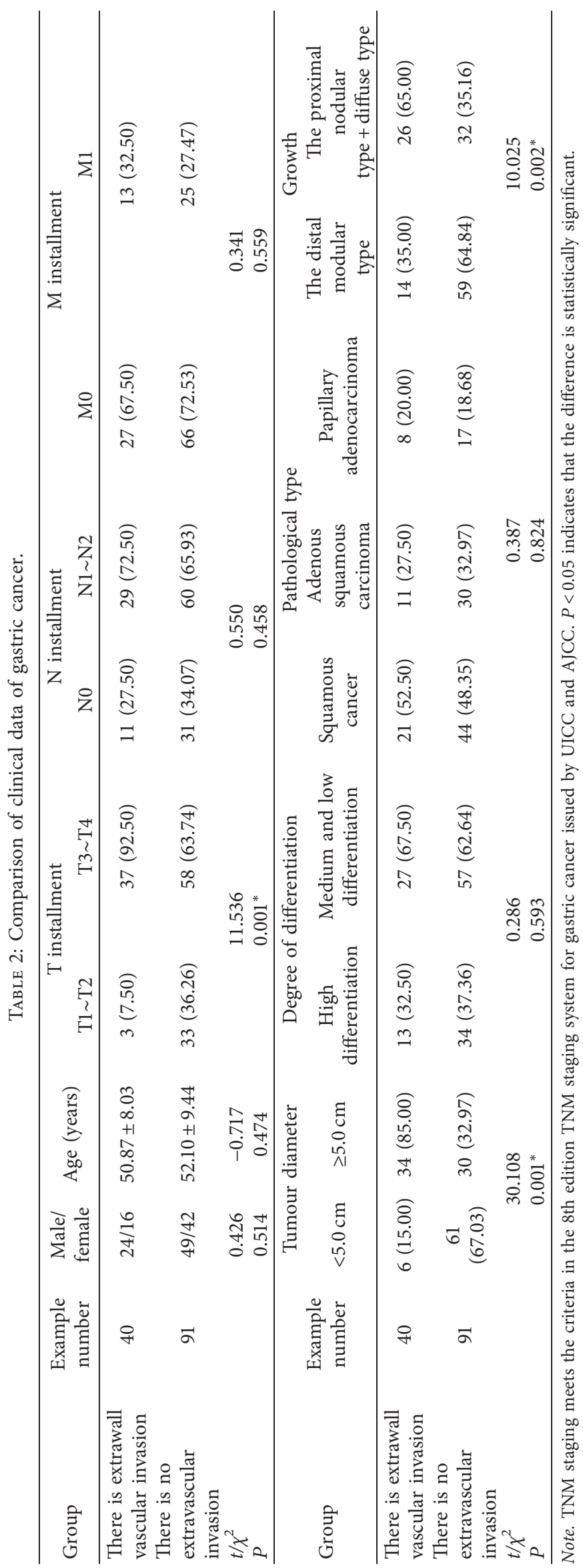


TABLE 3: Results of the logistic regression analysis.

\begin{tabular}{lccccr}
\hline Factors & $\beta$ & SE & Walds & $P$ & OR (95\%CI) \\
\hline T3 T4 & 1.322 & 0.324 & 16.648 & $0.001^{*}$ & $3.751(1.988 \sim 7.078)$ \\
Tumor diameter was $\geq 5.0 \mathrm{~cm}$ & 1.065 & 0.305 & 12.193 & $0.001^{*}$ & $2.901(1.596 \sim 5.274)$ \\
The proximal nodular type + diffuse type & 1.214 & 0.411 & 8.725 & $0.001^{*}$ & $3.367(1.504 \sim 7.535)$ \\
\hline
\end{tabular}

$P<0.05$ indicates that the difference is statistically significant.

the patient survival rate $[6,7]$. Currently, the standard diagnosis methods in China are still pathologically diagnosed by experienced doctors [8]. However, due to different experiences, sometimes, the misdiagnosis rate is high, affecting patient prognosis and reducing survival rate [9].

It has been shown that extrawall vascular invasion usually coexists with extraspinal nerve and lymphatic vessel infiltration and is a way of tumour spreading along the neurovascular tract [10]. Due to extrawall vascular invasion, tumour cells can spread throughout the portal system and eventually form distant metastasis [11]. The rate of distant metastasis in gastric cancer is positively associated with the degree of venous invasion, and external vascular invasion is an independent risk factor for predicting whether distant metastases occur and the chance of postoperative tumour recurrence [12-14]. Clinically, gene sequencing technology can classify gastric cancer into proximal nondiffuse type, distal nondiffuse type, and diffuse type, and distal nondiffuse type prognosis is significantly better than proximal nondiffuse type and diffuse gastric cancer [15]. The occurrence of extrawall vascular invasion in gastric cancer is closely related to the poor prognosis in gastric cancer patients. It is an independent risk factor for predicting distant metastasis and recurrence [16]. Surgical pathological diagnosis is an effective method to find extrawall vascular invasion, which can detect positive extravascular infiltration, but the results are susceptible to multiple factors and have specific false-negative effects [17].

This study creatively uses medical techniques and CT imaging techniques to diagnose extravascular invasion, and using CT imaging diagnosis can significantly improve diagnostic accuracy [18]; the diagnosis can be seen, and the images can be used to analyze the lesions as well as more intuitively display the diffusion situation, and the stability is improved [19], which is also the innovation of this study. After surgical pathological diagnosis, 40 patients showed external vascular invasion of gastric cancer [20].

In this study, $\mathrm{T}$ stage, tumour diameter, and growth mode were taken as independent variables, and whether extravascular invasion occurred was taken as the dependent variable for logistic regression analysis. The results showed that T3 T4, tumour diameter $\geq 5.0 \mathrm{~cm}$, and proximal nodular + diffuse growth mode were risk factors for extravascular invasion in gastric cancer. The diagnosis of ceMDCT for external vascular invasion in gastric cancer was associated with $\mathrm{T}$ stage, tumour growth mode, tumour size, and pathological stage.

The ceMDCT diagnosis of gastric cancer nonwalled vascular invasion and surgical pathological results were excellent. Its diagnostic sensitivity, specificity, positive predictive value, and negative predictive values were at high levels, and T3 T4, patients with gastric cancer tumour diameter $\geq 5.0 \mathrm{~cm}$, and proximal nodular + diffuse growth mode were significantly higher than in patients with nonmural vascular invasion. The analysis is that extravascular invasion of the tumour is based on the spread of the tumour along the nerve vessels. Thus, the larger the tumour, the greater the likelihood of proximal nodular and dispersal vascular invasion. It is shown that abdominal CT examination can be used to determine vascular invasion outside gastric cancer, which can more accurately visualize the patient's lesion and spread by image analysis and accurately observe the extent of tumor invasion and distant organs and lymph node metastases, helping to obtain a clearer diagnostic result.

This study is limited to retrospective imaging characterization, and prospective studies are needed to further determine the relevance of extramural vascular invasion with patient prognosis. The diagnosis of extrawall vessel invasion by preoperative ceMDCT would be an effective indicator of preoperative gastric cancer risk stratification after the TNM stage.

\section{Conclusions}

In conclusion, ceMDCT has good application value in the diagnosis of vascular invasion outside the gastric cancer wall, where vascular invasion is affected by $\mathrm{T}$ stage, tumour diameter, and tumour growth mode, and prospective studies are needed to further determine the relevance of extramural vascular invasion with patient prognosis.

\section{Data Availability}

The data used to support this study are available from the corresponding author upon request.

\section{Conflicts of Interest}

The authors declare no conflicts of interest.

\section{References}

[1] H. Liang, C. Li, and I. M. E. I. Zhang, "Study on efficacy and safety of neoadjuvant chemotherapy for treatment of locally advanced gastric cancer," Cancer progression, vol. 16, no. 3, pp. 345-348, 2018.

[2] F. Caizhen, C. Jin, and H. Xiang, "The predictive value of multirow CT for disease-free survival after gastric cancer," Chinese Journal of General Surgery, vol. 21, no. 9, pp. 10591064, 2018. 
[3] M. Yu, Y. Yu, and S. Zhang, "Value of multilayered helical CT for Lauren typing of progressive gastric cancer," Chinese Journal of Clinical Medical Imaging, vol. 28, no. 9, pp. 633636, 2017.

[4] Q. tied Wang and X. Zhao, "The diagnostic value of enhanced scan of layer 64 multicoiled CT for lymph node metastasis in preoperative gastric cancer," PLA Journal of Medicine, vol. 030, no. 010, pp. 27-30, 2018.

[5] S.-X. Yang, X. Yao, X.-H. Song, Y.-C. Cui, Y.-J. Ye, and Y. Wang, "Extramural vascular invasion detected by contrastenhanced multiple-row detectors computed tomography (ceMDCT) as a predictor of synchronous metastases in colon cancer," Oncotarget, vol. 8, no. 55, pp. 94883-94892, 2017.

[6] X. L. Wen, L. Wang, X. Q. Song, Z. Zhi, Z. Xiao Fang, and W. Shao Ping, "Clinical value of multi-slice spiral enhanced CT in diagnosing mild digestive tract hemorrhage," Zhongguo Yi Xue Ke Xue Yuan Xue Bao Acta Academiae Medicinae Sinicae, vol. 40, no. 2, pp. 139-140, 2018.

[7] T. U. Kim, S. Kim, N. K. Lee et al., "Prognostic value of computed tomography-detected extramural venous invasion to predict disease-free survival in patients with gastric cancer," Journal of Computer Assisted Tomography, vol. 41, no. 3, pp. 430-436, 2017.

[8] J. Liu, H. Fan, and G. P. Qiu, "Vascular permeability determined using multi-slice spiral CT perfusion can predict response to chemoradiotherapy in patients with advanced cervical squamous cell carcinoma," International Journal of Clinical Pharmacology \& Therapeutics, vol. 55, no. 7, pp. 619-626, 2017.

[9] M. R. S. Siddiqui, C. Simillis, C. Hunter et al., "A meta-analysis comparing the risk of metastases in patients with rectal cancer and MRI-detected extramural vascular invasion (mrEMVI) vs mrEMVI-negative cases," British Journal of Cancer, vol. 116, no. 12, pp. 1513-1519, 2017.

[10] S.-q. Wang, F.-y. Ren, J.-h. Wang et al., "Diagnostic value of multislice spiral computed tomography (CT) combined with CT angiography for intra-abdominal undescended testis secondary seminomas," Cancer Imaging, vol. 19, no. 1, pp. 24-25, 2019.

[11] L. G. J. Leijssen, A. M. Dinaux, R. Amri et al., "Impact of intramural and extramural vascular invasion on stage II-III colon cancer outcomes," Journal of Surgical Oncology, vol. 119, no. 6, pp. 749-757, 2019.

[12] J. S. Williamson, H. G Jones, N Williams et al., "Extramural vascular invasion and response to neoadjuvant chemoradiotherapy in rectal cancer: influence of the $\mathrm{CpG}$ island methylator phenotype," World Journal of Gastrointestinal Oncology, vol. 9, no. 5, pp. 209-217, 2017.

[13] E. E. van Eeghen, M. J. Flens, M. M. R. Mulder, and R. J. L. F. Loffeld, "Extramural venous invasion as prognostic factor of recurrence in stage 1 and 2 colon cancer," Gastroenterology Research and Practice, vol. 2017, no. 7, pp. 1-6, 2017.

[14] C. J. Streutker, "Extramural venous invasion in patients with locally advanced esophageal cancer: a reminder to pathologists to look harder," Annals of Surgical Oncology, vol. 25, no. 5, pp. 1465-1466, 2018.

[15] W. Chen, J. Gao, and D. Chen, "Guiding values of multislice spiral computed tomography angiography in laparoscopic D2 radical gastrectomy of local advanced gastric carcinoma," Journal of Cancer Research and Therapeutics, vol. 14, no. 8, pp. S197-S198, 2018.

[16] E. S. Lee, M. J. Kim, S. C. Park et al., "Magnetic resonance imaging-detected extramural venous invasion in rectal cancer before and after preoperative chemoradiotherapy: diagnostic performance and prognostic significance," European Radiology, vol. 28, no. 2, pp. 1-10, 2017.

[17] J. Li, W. Gao, B. Yu, F Wang, and L Wang, "Multi-slice spiral CT evaluation of breast cancer chemotherapy and correlation between CT results and breast cancerspecific gene 1," Journal of B.U.ON.: Official Journal of the Balkan Union of Oncology, vol. 23, no. 2, pp. 378-383, 2018.

[18] Z. Y. Xiao, H. J. Wang, C. L. Yao et al., "[Relationship between multi-slice spiral CT angiography imaging features and inhospital death of patients with aortic dissection]," Zhonghua Xinxueguanbing Zazhi, vol. 45, no. 3, pp. 217-218, 2017.

[19] J. Cheng, J. Wu, Y. Ye, C. Zhang, Y. Zhang, and Y. Wang, "Extramural venous invasion detected by MDCT as an adverse imaging feature for predicting synchronous metastases in T4 gastric cancer," Acta Radiologica, vol. 58, no. 4, pp. 387-393, 2017.

[20] G.-S. Jia, G.-L. Feng, J.-P. Li et al., "Using receiver operating characteristic curves to evaluate the diagnostic value of the combination of multislice spiral CT and alpha-fetoprotein levels for small hepatocellular carcinoma in cirrhotic patients," Hepatobiliary and Pancreatic Diseases International, vol. 16, no. 3, pp. 303-309, 2017. 